\title{
Accelerating your research career with Open Science
}

\author{
Dr Emma Norris \\ Department of Health Sciences; Brunel University London, UK \\ emma.norris@brunel.ac.uk
}

This is a preprint of the following chapter: Emma Norris, Accelerating your research career with Open Science, to be published in the upcoming book 'Survival Guide for Early Career Researchers', edited by Alden Lai and Dominika Kwasnicka, published by Springer Nature.

Preprint published according to Author's Self-Archiving Rights permitted within the Publishing Agreement with Springer Nature

\begin{abstract}
We could all benefit from our research and the research of others being more transparent and accessible to all, regardless of our career stage or research field. 'Open Science' provides us with a range of research practices designed to make the processes, data, analysis and outputs more reproducible, understandable and accessible. These practices range from making the protocol of your research explicit and publicly available via study pre-registration or Registered Report, to making a final published paper freely available via Open Access publishing or pre-print. This chapter will outline issues and challenges in research that Open Science helps to reduce. It will outline what Open Science is and how it can be integrated into your research workflow and summarise some global initiatives to increase Open Science uptake. The chapter will end by discussing how Open Science can benefit and accelerate your career and give you some tips on how to get started.
\end{abstract}


Research is never straight-forward. As you will have experienced, there are challenges underpinning each step of the research process. These challenges include how to define clear research aims and hypotheses, selecting appropriate methodologies, analysing complex data, negotiating collaboration with colleagues perhaps online and internationally: the list goes on! What are examples of problems you've had in your research so far?

I'll start by sharing (some!) of my own challenges. Systematic reviews form an important part of my work. As anyone who's done a review or meta-analysis will know, the process of searching for papers, filtering them for relevance, extracting data on them, synthesising their data is hugely time-intensive (Borah et al. 2017). One major barrier to fully completing a systematic review is not being able to access the raw data you need from past studies to make conclusions on the collective state of play on a topic. Or perhaps you want to get hold of a developed tool, intervention or questionnaire that you've read about but struggle to find who to ask? I've had many instances where I've popped a friendly email to a corresponding author asking for a data file, a few data values or some details about a study and received no response. There are obvious explanations for this; people move institutions and become difficult to locate, time passes since the study and the study data and materials may become lost or unmanaged. In extreme cases, the reported results may be fraudulent and the data may never have existed! How can we organise science better to ensure data is made routinely publicly available for us to easier learn from each other, as well as facilitate reviews and adoption of our work?

Another challenge I'm sure we've all experienced previously is the long lead-time between finishing a study, getting it peer-reviewed and published. I work in public health and health psychology, where I hope my work has implications for real-world practice and healthcare service delivery. From an early career researcher perspective, we need publications to put us in more favourable positions for job and grant applications. For those of us within academia, we operate in systemic problematic incentive structures where volume of papers and impact factors are primarily valued for recruitment and promotion, rather than research rigour and quality (Higginson \& Munafò, 2016). As such, the sometimes multiple year delay in publication of research is not helpful. How can we mitigate delays prominent in the peer-review process?

An additional challenge I've experienced as a researcher is coming into projects partway through, as a Research Assistant or Collaborator. It can be difficult to get to grips with what's been done so far, adding time to the familiarisation process. How can we better organise our work to make its processes more routinely transparent and understandable? 
I experienced these challenges primarily during my PhD. Now four years post-PhD, I am immersed in Open Science practices within my work which help mitigate these challenges for me. In this chapter I will attempt to convince you that research practices typically referred to as 'Open Science' practices can help tackle your own research challenges and make your research even more rewarding!

\section{What is Open Science?}

Open Science, also often referred to as Open Research, is a growing global movement aiming to make all aspects of research, education and scholarship accessible, transparent and available to all audiences. Open Science involves scientists, funding bodies and universities working collaboratively to increase reproducibility and transparency in the scientific process, reporting and teaching (Norris \& O'Connor, 2019; Pontika et al. 2015). The drive towards these more transparent practices is inpart driven by findings that research across disciplines is often not replicable. For example, a survey by the journal Nature of 1,576 researchers identified that more than $70 \%$ of surveyed researchers across disciplines had tried and failed to reproduce the findings of others, and more than $50 \%$ had failed to reproduce their own (Baker, 2016). Within psychology, a mass replication attempt of 100 experimental and correlational studies in social and cognitive psychology found that only $36 \%$ of replicated studies had statistically significant $(p<0.05)$ results, compared to $97 \%$ of the original studies (Open Science Collaboration, 2015).

Academia is rife with problematic incentive structures. Journal publications are often easier for novel and exciting positive findings rather than null or replicative findings (Edwards \& Roy, 2017). Promotion and recruitment criteria are commonly based on number of publications and Impact Factor of journals, rather than the actual tacit quality of the work (Nosek et al. 2012). The Journal Impact Factor, as calculated by Thomson Reuters, was originally created as a tool to help librarians identify journals to purchase, not as a measure of the scientific quality of research in an article. These problematic incentives help to drive research error and misconduct. For example, 'questionable research practices' such as selective reporting of outcomes (John et al., 2012) or hypothesizing after the results are known (HARKing; Kerr, 1998) are conscious researcher decisions, driven by the need for significant and publishable findings. High-profile cases of uncovered fraudulent researcher behaviour driven by the need for publication success have also driven this need for reform (Ritchie, 2020). For example, Diederik Stapel, former professor of social psychology at Tilburg University in the Netherlands was found to have manipulated and completely fabricated 
entire experiments resulting in at least 30 published, peer-reviewed papers (Markowitz \& Hancock, 2014).

A range of Open Science reforms to scientific practice have been installed over recent years to increase the reproducibility and transparency of scientific practice. These include specific research practices that can be integrated throughout the research process (Figure 1): before, during and after data collection. For example, research 'preregistration' is the practice of submitting a research study plan to an online repository (such as Open Science Framework: Sullivan et al. 2019) prior to the research being undertaken (Hardwicke \& Wagenmakers, 2021; Lakens, 2019). Preregistration aims to mitigate against questionable research practices and increase transparency (Nosek et al., 2018; O'Connor et al., 2021) by pre-specifying all hypotheses, methods and analysis plans and reduced unnoticed flexibility (Nosek et al. 2019). It is hoped that with greater transparency comes greater rigour, and so greater credibility of research (Field et al., 2020; Ihle et al., 2017). Preregistration of research is also intended to reduce the 'file drawer' problem (O'Connor, 2021), whereby negative and null findings are less likely to be published than positive, statistically significant findings (Rosenthal, 1979).

Figure 1. Examples of Open Science practices across the research process

\begin{tabular}{|c|c|}
\hline Open Science practice & Definition and example \\
\hline \multicolumn{2}{|l|}{ Before data collection } \\
\hline Preregistration & $\begin{array}{l}\text { Submitting a research study plan to an online repository prior to } \\
\text { the research being undertaken. (Field et al. 2020; Lakens, 2019). } \\
\text { Example: Open Science Framework https://osf.io and AsPredicted } \\
\text { https://aspredicted.org provide pre-registration templates and } \\
\text { registrations. }\end{array}$ \\
\hline Registered Reports & $\begin{array}{l}\text { A form of journal article with a 2-stage peer-review and } \\
\text { acceptance process. In Stage 1, the proposed methods and } \\
\text { analyses are pre-registered and peer-reviewed prior to research } \\
\text { being conducted. High quality protocols are then provisionally } \\
\text { accepted for publication before data collection commences. In } \\
\text { Stage 2, once the study is completed, the author will finish the } \\
\text { article including results and discussion sections for this to be peer- } \\
\text { reviewed. (Chambers, \& Tzavella, 2020; Stewart et al. 2020). } \\
\text { Example: Many journals accepting Registered Reports can be } \\
\text { found within the Peer Community In (PCI) Registered Reports } \\
\text { scheme: https://rr.peercommunityin.org/about/about }\end{array}$ \\
\hline
\end{tabular}




\begin{tabular}{|l|l|}
\hline \multicolumn{2}{|l|}{ During and after data collection } \\
\hline Open Data & $\begin{array}{l}\text { Data that can be freely used, re-used and redistributed by anyone } \\
\text { - subject only, at most, to the requirement to attribute and } \\
\text { sharealike. } \\
\text { Example: FAIR data principles require data to meet the principles } \\
\text { of findability, accessibility, interoperability, and reusability. } \\
\text { (Wilkinson et al. 2016) }\end{array}$ \\
\hline Open Materials & $\begin{array}{l}\text { Publishing materials used within research, such as experiments, } \\
\text { tasks, questionnaires or intervention content. } \\
\text { Example: Journals increasingly offer badges to reward publication } \\
\text { of Open Materials alongside papers, as well as for Open Data and } \\
\text { Pre-Registration (Kidwell et al., 2016) }\end{array}$ \\
\hline Open-Source Software & $\begin{array}{l}\text { Computer software released under a licence in which the } \\
\text { copyright holder permits users the rights to use, change and } \\
\text { distribute the software and its source code (Fortunato \& Galassi, } \\
\text { 2021). } \\
\text { Example: R software for statistical computing https://www.r- } \\
\text { project.org/ }\end{array}$ \\
\hline Publication & $\begin{array}{l}\text { Version of a scholarly paper that is made publicly available online } \\
\text { prior to formal peer review and publication in a peer-reviewed } \\
\text { journal. } \\
\text { Example: OSF Pre-prints offers pre-print services by research } \\
\text { subjects https://osf.io/preprints/ }\end{array}$ \\
\hline Pre-prints & $\begin{array}{l}\text { Making research publications freely available so anyone can read } \\
\text { and use them. } \\
\text { Example: Sherpa Romeo allows you to check the open access } \\
\text { policies in journals globally https://v2.sherpa.ac.uk/romeo/ }\end{array}$ \\
\hline $\begin{array}{l}\text { Scholarly review providing disclosure of author and referee } \\
\text { identifies during the peer review process and published alongside } \\
\text { the resultant article (Ross-Hellauer, 2017). } \\
\text { Example: Wellcome Open Research } \\
\text { https://wellcomeopenresearch.org/ }\end{array}$ \\
\hline
\end{tabular}

Note: See Kathawalla et al. (2021) for a great introductory guide on how to integrate Open Science across the research process as an early career researcher.

\section{Global initiatives to increase Open Science}

International efforts to facilitate Open Science are ever-increasing. As early career researchers, we are set to benefit from these current and future reforms to the academic ecosystem as we progress in our careers. One development which has provided us all with an infrastructure to work more openly is Open Science Framework (OSF: Foster \& Deardorff, 2017): https://osf.io. OSF provides a 
free platform to create pages for each of our projects: allowing us to preregister the research, share the data and materials and publish preprints all in one place. It was developed and is maintained by the Center for Open Science (COS), a non-profit organization founded in $2013 \mathrm{https}: / /$ cos.io/. We can all benefit from OSF by making our own research more accessible but also by being able to search for related work globally and establish new collaborations from this. Global strategies are also working to reduce aforementioned problematic incentive structures within academia. The San Francisco Declaration on Research Assessment (DORA) principles aims to prevent the practice of correlating journal impact factor with scientific merit. It is an opt-in agreement that universities and other institutions can opt-in to which states they will not use impact factor as a substitute "measure of the quality of individual research articles, or in hiring, promotion, or funding decisions" (Alberts, 2013).

The Transparency and Openness Promotion (TOP) guidelines offer standards as a basis for journals and funders to incentivise or require greater transparency in planning and reporting of research (McNutt, 2016). The TOP guidelines include eight modular standards encompassing Data citation, Data, Materials and Code Transparency, Design and Analysis, Preregistration and Replication, each with three levels of increasing stringency: Disclosure, Require or Verify. Journals select which of the eight transparency standards they wish to implement and select a level of implementation for each (Nosek et al. 2016). This provides clarity for us as authors in the degree of specificity and transparency required ahead of each journal submission, reducing ambiguity. Academic reporting checklists also provide us with structured guidance on how to adequately report all aspects of our research in publications. The EQUATOR Network provides a suite of over 400 developed reporting tools across study designs, disciplines and populations (Altman \& Simera, 2016). For example, the Consolidated Standards of Reporting Trials (CONSORT) statement provides guidance for clear, complete and accurate reporting of randomized controlled trials (Schulz et al. 2010). By following appropriate checklists for our work, we can be sure to integrate all essential features within our research protocol development and write-up.

\section{How can Open Science help your career?}

Working in a more Open way is clearly beneficial to our understanding and appraisal of science at a global level. However, you'll likely find that upskilling yourself in various aspects of Open Science takes time and adds extra considerations to the research process (Allen \& Mehler, 2019; McKiernan et al., 2016). You may see working more openly as admirable but what are the benefits to you as an 
individual early career researcher? I'll now seek to sell the benefits of Open Science to you from my own experiences. These benefits are informed by the fantastic article by Florian Markowetz 'Five selfish reasons to work reproducibly' (Markowetz, 2015).

- Open Science helps to avoid disaster

Have you ever published a paper, been asked some details about the results and found that you've been unable to replicate your own findings? Have you looked back at your own analysis 6 months, a year or more down the line and totally forgotten how and why you analysed the data previously? You're not alone! Getting into the routine of logging decisions around your data's structure and analysis whilst you're working on it is beneficial to you in the long-run. Thorough decision-tracking is also beneficial as you work towards managing your own projects. In my own work, keeping a clear decision log of reasons behind paper inclusions and exclusions has been essential for studies involving multidisciplinary teams based in different countries. For quantitative research, keeping clear notes and tracking decisions by logging detailed notes in your syntax or using the R package workflowr (Bilschak et al. 2019) is a great idea. However simple methods are also useful, such as constructing and maintaining a shared Word or Google document of decisions for yourself or your team. For qualitative research, detailed decision logs can help reduce ambiguity in the themes you identify to yourself and other people later on.

- Open Science helps reviewers see it your way

Peer reviewers are becoming increasingly prepared to see data, a preregistration or a detailed Open Science Framework page to accompany papers they review. This allows them to better appraise the quality and transparency of your work. Providing additional detail like this allows you to tell a clearer story of your research: filling in the gaps that a 3,000 word journal article just doesn't have space for. Additionally, the increase in Open Peer Review means that reviewers can appraise your experience in-line with the research you have submitted (Wolfram et al. 2020).

- Open Science helps to build your reputation

For me, using and speaking about Open Science practices within public health and health behaviour change has opened a range of opportunities: such as writing this book chapter! Firstly, being open about your use of Open Science can grow your reputation in your existing institution. Although use of Open Science is on the increase, uptake in many disciplines is still relatively low and varies highly between institutions. I work in a Public Health department where understanding and uptake of Open Science is currently low. Showing an interest and being an advocate for Open Science has enabled 
me to start conversations across my own university on how to develop tailored initiatives: all beneficial for future promotion attempts! Secondly, being engaged and active in Open Science can lead to fantastic collaborations. For example, I am a co-Chair of the European Health Psychology Society's Open Science Special Interest Group (Norris \& Toomey, 2020), which involves developing initiatives to increase Open Science with colleagues from across Europe. Members of the Group have quite diverse research interests and so working together to promote Open Science provides a legitimate and important reason for collaboration which is not tied down to a specific research project.

- Open Science helps you build your research competence

Perhaps most importantly, putting extra thought into Open Science considerations has made me personally feel increasingly competent as a researcher. I completed my PhD in January 2017 and was totally unaware of Open Science at this time beyond Open Access publishing and Open Data. Emerging from a PhD where I was supervised by four multidisciplinary experts, I felt nervous about being able to maintain quality of research under my own steam. However, immersing myself in Open Science has enabled me to increase my confidence as an independent and thorough researcher. For example, the practice of preregistering a study plan before data collection has enabled me to be far more detailed and explicit in study design and analysis plans than I was before, reducing ambiguity when dealing with the data subsequently. Additionally, grant funders and journals are increasingly requiring Data Management Plans and evidence of Open Science prior to submission. It is reassuring to think that Open Science is now integrated into my workflow, and I can now concentrate on the content and scientific contribution of the research itself.

\section{Conclusions and recommendations}

As we've seen, a range of Open Science practices have been established across academic disciplines in recent years to improve the transparency and reproducibility of research. Using principles of Open Science is the present and future of best research practice. As early career researchers, we have much to benefit from these initiatives in terms of contributing to and spearheading higher quality scientific research and increasing our own research competence. Looking at the range of Open Science practices that exist (Figure 1 provides a snapshot of these) can be somewhat daunting. My one top tip is to not try and integrate all of these Open Science practices in any given project! Try and integrate one new Open Science aspect into each new project. For example, add a pre- 
registration or try publishing a preprint. Take time to evaluate how you found your experience and consider if you'll seek to improve this or try another practice in your next project.

I'll end with one of my favourite Open Science quotes from the outstanding Open Science advocate and co-founder of ReproducibiliTea Open Science Journal Clubs Dr Amy Orben from University of Cambridge, UK: “Open science is a process, not a one-time achievement or a claim to virtue" (Orben, 2019). Open Science is not a virtue signalling exercise. It serves both as for the collective benefit for science, as well as for improved personal confidence and competent in science. Open Science is here to stay: Get involved!

\section{References}

Alberts, B. (2013). Impact factor distortions. Science, 787.

Allen, C., \& Mehler, D. M. (2019). Open science challenges, benefits and tips in early career and beyond. PLoS Biology, 17(5), e3000246.

Altman, D. G., \& Simera, I. (2016). A history of the evolution of guidelines for reporting medical research: the long road to the EQUATOR Network. Journal of the Royal Society of Medicine, 109(2), 67-77.

Baker, M. (2016). 1,500 scientists lift the lid on reproducibility. Nature, 533(26), 353-

66. https://doi.org/10.1038/533452a

Blischak, J. D., Carbonetto, P., \& Stephens, M. (2019). Creating and sharing reproducible research code the workflowr way. F1000Research, 8.

Borah, R., Brown, A. W., Capers, P. L., \& Kaiser, K. A. (2017). Analysis of the time and workers needed to conduct systematic reviews of medical interventions using data from the PROSPERO registry. BMJ Open, 7(2), e012545.

Chambers, C., \& Tzavella, L. (2020). Registered reports: Past, present and future. https://doi.org/10.31222/osf.io/43298

Edwards, M. A., \& Roy, S. (2017). Academic research in the 21st Century: Maintaining scientific integrity in a climate of perverse incentives and hypercompetition. Environmental Engineering Science, 34(1), 51-61. https://doi.org/10.1089/ees.2016.0223

Field, S. M., Wagenmakers, E. J., Kiers, H. A., Hoekstra, R., Ernst, A. F., \& van Ravenzwaaij, D. (2020). The effect of preregistration on trust in empirical research findings: Results of a registered report. Royal Society Open Science, 7(4), 181351. https://doi.org/10.1098/rsos.181351 
Fortunato L, Galassi M. (2021). The case for free and open source software in research and scholarship. Philosophical Transactions of the Royal Society A Mathematical, Physical and Engineering Sciences ;379(2197):20200079. https://doi.org/10.1098/rsta.2020.0079 Foster, E. D., \& Deardorff, A. (2017). Open science framework (OSF). Journal of the Medical Library Association, 105(2), 203.

Hardwicke, T. E., Thibault, R. T., Kosie, J., Wallach, J. D., Kidwell, M. C., \& loannidis, J. (2021). Estimating the prevalence of transparency and reproducibility-related research practices in psychology (2014-2017). Perspectives on Psychological Science, https://doi.org/10.1177\%2F1745691620979806

Higginson, A. D., \& Munafò, M. R. (2016). Current incentives for scientists lead to underpowered studies with erroneous conclusions. PLOS Biology, 14(11), e2000995. https://doi.org/10.1371/journal.pbio.2000995 John, L. K., Loewenstein, G., \& Prelec, D. (2012). Measuring the prevalence of questionable research practices with incentives for truth telling. Psychological Science, 23(5), 524-

532. https://doi.org/10.1177/0956797611430953

Kathawalla, U. K., Silverstein, P., \& Syed, M. (2021). Easing into open science: A guide for graduate students and their advisors. Collabra: Psychology, 7(1).

Kerr, N. L. (1998). HARKing: Hypothesizing after the results are known. Personality and Social Psychology Review, 2(3), 196-217.

Kidwell, M. C., Lazarević, L. B., Baranski, E., Hardwicke, T. E., Piechowski, S., Falkenberg, L. S., ... \& Nosek, B. A. (2016). Badges to acknowledge open practices: A simple, low-cost, effective method for increasing transparency. PLoS Biology, 14(5), e1002456. https://doi.org/10.1371/journal.pbio.1002456 Markowetz, F. (2015). Five selfish reasons to work reproducibly. Genome Biology, 16(1), 1-4. Markowitz, D. M., \& Hancock, J. T. (2014). Linguistic traces of a scientific fraud: The case of Diederik Stapel. PloS One, 9(8), e105937.

McKiernan, E. C., Bourne, P. E., Brown, C. T., Buck, S., Kenall, A., Lin, J., McDougall, D., Nosek, B. A., Ram, K., Soderberg, C. K., Spies, J. R., Thaney, K., Updegrove, A., Woo, K. H., \& Yarkoni, T. (2016). How open science helps researchers succeed. ELife, 5, e16800. https://doi.org/10.7554/eLife.16800 McNutt, M. (2016). Taking up TOP. Science, 352, 1147.

Munafò, M. R., Nosek, B. A., Bishop, D. V., Button, K. S., Chambers, C. D., du Sert, N. P., ... \& loannidis, J. P. (2017). A manifesto for reproducible science. Nature Human Behaviour, 1(1), 0021. https://doi.org/10.1038/s41562-016-0021 
Norris, E., \& O'Connor, D. B. (2019). Science as behaviour: Using a behaviour change approach to increase uptake of open science. Psychology \& Health, 34(12), 1397-1406.

Norris, E. \& Toomey, E. (2020). Open Science in Health Psychology: Launching the EHPS Open Science SIG. The European Health Psychologist. 21(5). 679-682.

Nosek, B. A., Alter, G., Banks, G. C., Borsboom, D., Bowman, S., Breckler, S., ... \& DeHaven, A. (2016). Transparency and openness promotion (TOP) guidelines. https://osf.io/vj54c

Nosek, B. A., Spies, J. R. \& Motyl, M. Scientific utopia: II. Restructuring incentives and practices to promote truth over publishability. Perspectives in Psychological Science, 7, 615-631 (2012).

Open Science Collaboration. (2015). Estimating the reproducibility of psychological science. Science, 349(6251).

Orben, A. (2019). A journal club to fix science. Nature, 573: 465.

Pontika N, Knoth P, Cancellieri M, et al (2015). Fostering open science to research using a taxonomy and an eLearning portal. Proceedings of the $15^{\text {th }}$ International Conference on Knowledge Technologies and Data-driven Business. Graz, Austria: Association for Computing Machinery. Ritchie, S. (2020). Science Fictions: Exposing Fraud, Bias, Negligence and Hype in Science. London: Penguin.

Ross-Hellauer, T. (2017). What is open peer review? A systematic review. F1000Research, 6. Schulz, K. F., Altman, D. G., \& Moher, D. (2010). CONSORT 2010 statement: updated guidelines for reporting parallel group randomized trials. Annals of Internal Medicine, 152(11), 726-732.

Stewart, S., Rinke, E. M., McGarrigle, R., Lynott, D., Lunny, C., Lautarescu, A., ... \& Crook, Z. (2020). Pre-registration and Registered Reports: A primer from

UKRN. https://osf.io/8v2n7/download?format=pdf

Tenopir, C., Rice, N. M., Allard, S., Baird, L., Borycz, J., Christian, L., ... \& Sandusky, R. J. (2020). Data sharing, management, use, and reuse: Practices and perceptions of scientists worldwide. PloS One, 15(3), e0229003. https://doi.org/10.1371/journal.pone.0229003 Wilkinson, M. D., Dumontier, M., Aalbersberg, I. J., Appleton, G., Axton, M., Baak, A., ... \& Mons, B. (2016). The FAIR Guiding Principles for scientific data management and stewardship. Scientific Data, 3(1), 1-9.

Wolfram, D., Wang, P., Hembree, A., \& Park, H. (2020). Open peer review: promoting transparency in open science. Scientometrics, 125, 1033-1051. 